\title{
Prognostic utility of neutrophil-to-lymphocyte ratio in patients with metastatic colorectal cancer treated using different modalities
}

\author{
G. Nogueira-Costa MD,* I. Fernandes MD, ${ }^{*}$ R. Gameiro MD,* J. Gramaça MD,* A.T. Xavier MD,* \\ and I. Pina MD*
}

\begin{abstract}
Introduction Inflammation is a critical component in carcinogenesis. The neutrophil-to-lymphocyte ratio (NLR) has been retrospectively studied as a biomarker of prognosis in metastatic colorectal cancer (mCRC). Compared with a low NLR, a high NLR is associated with worse prognosis. In the present study, we compared real-world survival for patients with mCRC based on their NLR group, and we assessed the utility of the NLR in determining first-line chemotherapy and metastasectomy benefit.
\end{abstract}

Methods In this retrospective and descriptive analysis of patients with mCRC undergoing first-line chemotherapy in a single centre, the last systemic absolute neutrophil and lymphocyte count before treatment was used for the NLR. A receiver operating characteristic curve was used to estimate the NLR cut-off value, dividing the patients into low and high NLR groups. Median overall survival (mOS) was compared using Kaplan-Meier curves and the log-rank test. A multivariate analysis was performed using a Cox regression model.

Results The 102 analyzed patients had a median follow-up of 15 months. Regardless of systemic therapy, approximately $20 \%$ of patients underwent metastasectomy. The NLR cut-off was established at 2.35 , placing 45 patients in the low-risk group (NLR <2.35) and 57 in the high-risk group (NLR $\geq 2.35)$. The Kaplan-Meier analysis showed a mOS of 39.1 months in the low-risk group and 14.4 months in the high-risk group $(p<0.001)$. Multivariate Cox regression on the NLR estimated a hazard ratio of $3.08(p=0.01)$. Survival analysis in each risk subgroup, considering the history of metastasectomy, was also performed. In the low-risk group, mos was longer for patients undergoing metastasectomy than for those not undergoing the procedure (95.2 months vs. 22.6 months, $p=0.05$ ). In the high-risk group, mos was not statistically different for patients undergoing or not undergoing metastasectomy (24.3 months vs. 12.7 months, $p=0.08$ ).

Conclusions Our real-world data analysis of NLR in patients with mCRC confirmed that this biomarker is useful in predicting survival. It also suggests that NLR is an effective tool to choose first-line treatment and to predict the benefit of metastasectomy.

Key Words Colorectal cancer, metastatic; neutrophils; lymphocytes; NLR; metastasectomy

\section{INTRODUCTION}

Colorectal cancer (CRC) is the 2nd most common cancer in most developed countries, with an estimated incidence of $12.8 \%$ and a mortality of $12.6 \%$ in both sexes ${ }^{1}$. New evidence suggests that, at the time of diagnosis, up to $80 \%$ of patients with CRC might present with disseminated cells that seed metastasis ${ }^{2}$. Multiple factors that contribute to tumour spread have been identified, including tumour size, neurovascular or lymphatic invasion, resection margin, sidedness, molecular features, and inflammatory response $^{3-5}$. Indeed, the association between inflammation and carcinogenesis has been widely documented. The activation and proliferation of immune cells associated with a local microenvironment able to sustain specific conditions for tumour growth potentiates the risk of cancer 
development and progression ${ }^{6,7}$. Measurement of inflammatory markers has therefore shown promising results in predicting survival in patients with cancer ${ }^{8}$.

As in other solid tumours, carcinogenesis in CRC is linked to inflammation ${ }^{9,10}$. In recent years, many studies have analyzed survival with respect to the systemic inflammatory response and studied the applicability of predictive immune scores in CRC. Examples include the lymphocyte/ monocyte ratio, the platelet/lymphocyte ratio, and the modified Glasgow Prognostic Score, which is determined using serum C-reactive protein and serum albumin ${ }^{11,12}$. Another score that shows promising results in risk prediction is the neutrophil/lymphocyte ratio (NLR). Worse survival outcomes have been linked to patients with CRC who have a high NLR compared with a low NLR. That association has been confirmed at various stages of CRC, from early localized disease before surgical resection ${ }^{13,14}$ to more advanced stages $^{15-18}$. The NLR has also been studied in patients with CRC who had undergone liver metastasectomy ${ }^{19}$.

In the metastatic setting, guidelines recommend first-line treatment regimens of doublet or triplet fluoropyrimidine-based chemotherapy (CTx) plus a molecularly targeted agent. In addition, the decision about treatment intensity integrates therapeutic goals, usually considering the patient's clinical and radiologic features ${ }^{20}$. If conversion to resectable disease for an eventual surgical curative approach is the goal, a more-intensive regimen is proposed. Whenever disease control is the goal, a less-intensive CTx regimen is usually the first option to control disease progression while maintaining quality of life.

The purpose of the present study was to determine the NLR in real-world patients with mCRC and to compare overall survival (os) in risk-stratified groups based on the NLR. A secondary purpose was to determine if the NLR risk group could also be useful in selecting patients for firstline therapy for conversion to resectability and eventual metastasectomy.

\section{METHODS}

\section{Patients}

This retrospective cohort study considered consecutive patients with mCRC who were treated for at least 1 month with CTx during 2015-2017 at the Medical Oncology Unit of Centro Hospitalar Barreiro-Montijo, Portugal. Patients who received neoadjuvant or adjuvant therapy or who had history of emergency surgery for colon obstruction or perforation were also included in the analysis. All first-line CTx regimens were fluoropyrimidine-based with or without associated targeted therapy depending on $R A S$ mutation status. Baseline patient and tumour characteristics, evidence of metastasis at diagnosis, history of surgical resection of metastases, history of adjuvant treatment, first-line treatment, and response were all retrospectively collected from the hospital's electronic database and patient records. The last follow-up data were collected as of 28 February 2019.

\section{Blood-Sample Analysis}

Peripheral blood was collected during the week before cycle 1 of first-line CTx. The NLR was calculated from the counts obtained using that sample, dividing the absolute neutrophil count by the absolute lymphocyte count.

\section{Statistics}

Descriptive statistics are used to describe baseline characteristics and treatments. A receiver operating characteristic (ROC) curve was used to define the most accurate cut-off value for the NLR. Then, based on the cut-off value and their NLR, patients were allocated to one of two risk groups: high risk or low risk. The os duration was defined as the time from diagnosis of metastatic disease to death from any cause. Median os (mos) was estimated using the Kaplan-Meier method, and outcome parameters were compared using the log-rank test. A multivariate analysis performed according to the Cox regression model calculated hazard ratios. A significance level of $p<0.05$ was used for all tests. The IBM SPSS Statistics software application (version 24.0: IBM, Armonk, NY, U.S.A.) for Windows (Microsoft Corporation, Redmond, WA, U.S.A.) was used for all statistical analyses.

\section{RESULTS}

The study included 102 patients with mCRC treated between 1 January 2015 and 31 December 2017 at our institution. Median follow-up was 15 months (range: 1-106 months). Table I details patient demographics and baseline characteristics. Mean age at diagnosis of metastatic disease in this cohort was 68 years (range: $41-84$ years), and the male:female ratio was approximately $2: 1$ (63.7\% vs. $36.3 \%)$.

The mean neutrophil-to-lymphocyte ratio (NLR) was $3.06 \pm 1.91$, with a median of 2.5 and a range of $0.6-10.5$. The NLR cut-off in our cohort, obtained by ROC curve, was 2.35 (sensitivity, 69.0\%; specificity, 61.4\%; Figure 1). The NLR was 2.35 or higher in 57 patients (56\%, high-risk group); it was less than 2.35 in 45 patients ( $44 \%$, low-risk group).

The location of the primary tumour was most commonly the left side in both the high-risk group and the low-risk group. Approximately $60 \%$ of the patients had metastatic disease when diagnosed with CRC. Resection of the primary tumour was performed in $93.3 \%$ of the low-risk group and in $64 \%$ of the high-risk group. $R A S$ mutation was found to be present in $43.1 \%$ of the patients and absent in $36.3 \%$ ( $R A S$ wild-type). In the remaining patients, no information about $R A S$ mutational status was available. A doublet CTx regimen was given together with an anti-vascular endothelial growth factor antibody in $28.4 \%$ of cases and with an anti-epidermal growth factor receptor antibody in $22.5 \%$ of cases. One third of the cohort received a doublet regimen with no targeted agent. About $10 \%$ of patients received a fluoropyrimidine alone, and $5 \%$ received a fluoropyrimidine plus an anti-vascular endothelial growth factor antibody. One patient received a triplet regimen plus an anti-vascular endothelial growth factor antibody. Approximately $20 \%$ of the cohort underwent surgical resection of metastases after a favourable response to first-line CTx.

Median os by NLR risk group was estimated using the Kaplan-Meier method. In the low-risk group, mos was 39.1 months [95\% confidence interval (CI): 3.7 months to 74.4 months]; in the high-risk group, it was 14.4 months (95\% CI: 
TABLE I Baseline patient characteristics

\begin{tabular}{|c|c|c|c|}
\hline \multirow[t]{2}{*}{ Characteristic } & \multicolumn{3}{|c|}{ Neutrophil-to-lymphocyte ratio } \\
\hline & $\begin{array}{l}\text { Low } \\
(<2.35)\end{array}$ & $\begin{array}{c}\text { High } \\
(\geq 2.35)\end{array}$ & Overall \\
\hline Patients ( $n$ ) & 45 & 57 & 102 \\
\hline \multicolumn{4}{|l|}{ Age (years) } \\
\hline Median & 69 & 68 & 68 \\
\hline Range & $54-82$ & $41-84$ & $41-84$ \\
\hline \multicolumn{4}{|l|}{$\operatorname{Sex}(\%)$} \\
\hline Men & 57.8 & 68.4 & 63.7 \\
\hline Women & 42.2 & 31.6 & 36.3 \\
\hline \multicolumn{4}{|l|}{ Tumour sidedness (\%) } \\
\hline Left and rectum & 66.7 & 82.5 & 75.5 \\
\hline Right & 33.3 & 17.5 & 24.5 \\
\hline Metastasis at Dx (\%) & 51.1 & 66.7 & 59.8 \\
\hline Resection of primary (\%) & 93.3 & 64.9 & 77.5 \\
\hline Metastasectomy (\%) & 22.2 & 21.1 & 21.6 \\
\hline $\begin{array}{l}\text { Neoadjuvant or adjuvant } \\
\text { treatment }(\%)\end{array}$ & 33.3 & 28.1 & 30.4 \\
\hline \multicolumn{4}{|l|}{ Mutation status (\%) } \\
\hline$R A S$ mutation & 33.3 & 50.9 & 43.1 \\
\hline RAS wild-type & 40.0 & 33.3 & 36.3 \\
\hline Not available & 26.7 & 15.8 & 20.6 \\
\hline \multicolumn{4}{|l|}{ First-line CTx (\%) } \\
\hline Fluoropyrimidine & 15.6 & 7.0 & 10.8 \\
\hline $\begin{array}{l}\text { Fluoropyrimidine + } \\
\text { anti-VEGF }\end{array}$ & 2.2 & 7.1 & 4.9 \\
\hline Doublet CTx & 26.7 & 36.8 & 32.4 \\
\hline Doublet + anti-EGFR & 31.1 & 15.8 & 22.5 \\
\hline Doublet + anti-VEGF & 24.4 & 31.6 & 28.4 \\
\hline Triplet + anti-VEGF & 0 & 1.8 & 1 \\
\hline
\end{tabular}

$\mathrm{Dx}=$ diagnosis; $\mathrm{CTx}=$ chemotherapy; VEGF = vascular endothelial growth factor; EGFR = epidermal growth factor receptor.

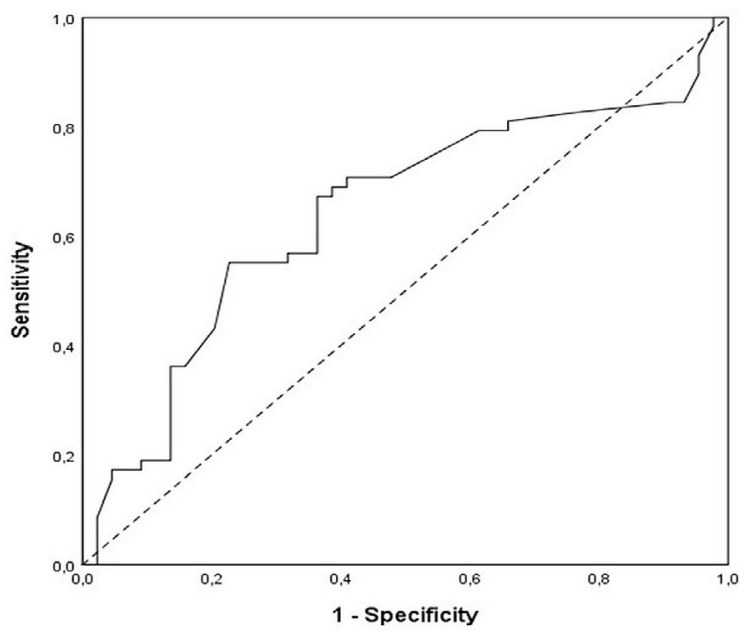

FIGURE 1 Receiver operating characteristic curve analysis of the neutrophil-to-lymphocyte ratio in patients with metastatic colorectal cancer. Area under the curve: $0.640 ; 95 \%$ confidence interval: 0.53 to $0.75 ; p=0.016$.
10.2 months to 18.6 months, $p<0.001$; Figure 2). Multivariate Cox regression including patient demographic and baseline characteristics resulted in a hazard ratio of 3.08 for the NLR variable (95\% CI: 1.35 to $7.01 ; p=0.01$; Table II).

In the low-risk NLR group, Kaplan-Meier os showed that patients whose metastases were surgically removed experienced a mos of 95.2 months (95\% CI: not reached to not reached) and that patients who did not receive surgery for metastasis experienced a mos of 22.6 months $(95 \%$ CI: 4.9 months to 40.2 months, $p=0.05$ ). In the high-risk group, patients receiving metastasectomy experienced a mos of 24.3 months (95\% CI: 8.0 months to 40.5 months), and those not receiving surgical intervention experienced an estimated mos of 12.7 months (95\% CI: 8.5 months to 16.8 months, $p=0.08$; Figure 3 ).

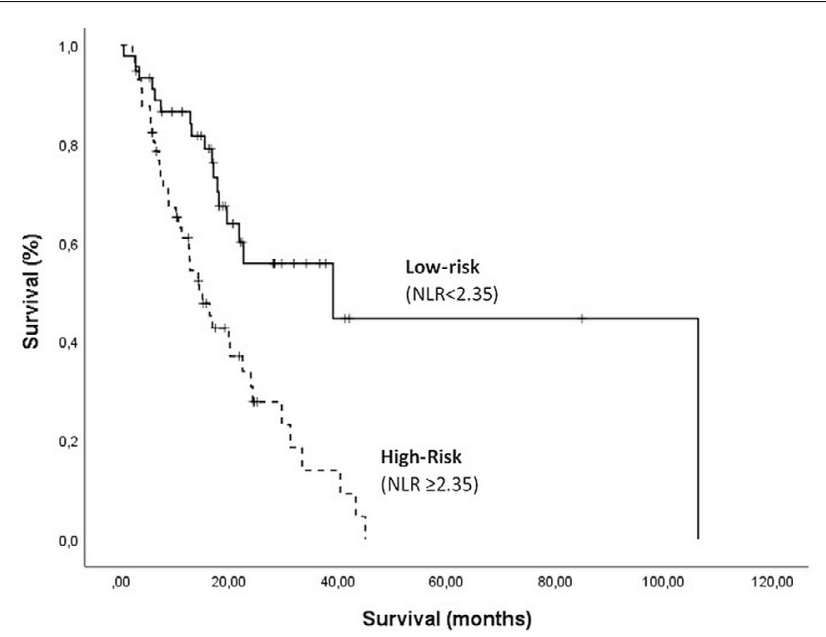

FIGURE 2 Kaplan-Meier survival analysis. Overall population survival was significantly worse in patients with a high neutrophil-to-lymphocyte ratio (NLR), $p=0.001$.

TABLE II Multivariate Cox regression analysis for cancer-specific survival and various clinicopathologic factors

\begin{tabular}{|c|c|c|c|}
\hline Variable & HR & $95 \% \mathrm{Cl}$ & $p$ Value \\
\hline Age & 1.02 & 0.98 to 1.07 & 0.29 \\
\hline Sex (men vs. women) & 0.72 & 0.35 to 1.48 & 0.37 \\
\hline $\begin{array}{l}\text { Tumour sidedness } \\
\text { (left vs. right) }\end{array}$ & 1.66 & 0.69 to 3.96 & 0.26 \\
\hline $\begin{array}{l}\text { Neoadjuvant or adjuvant } \\
\text { treatment }\end{array}$ & 1.66 & 0.52 to 5.33 & 0.39 \\
\hline Metastasis at diagnosis & 0.59 & 0.19 to 1.82 & 0.35 \\
\hline Resection of primary & 0.45 & 0.20 to 1.02 & 0.06 \\
\hline Metastasectomy & 0.34 & 0.15 to 0.79 & 0.01 \\
\hline $\begin{array}{l}\text { RAS mutation } \\
\quad \text { (mutated vs. wild-type) }\end{array}$ & 1.03 & 0.52 to 2.04 & 0.93 \\
\hline Received a targeted agent & 1.04 & 0.49 to 2.21 & 0.93 \\
\hline $\begin{array}{l}\text { NLR }[\geq 2.35 \\
\text { (high) vs. }<2.35 \text { (low)] }\end{array}$ & 3.08 & 1.35 to 7.01 & 0.01 \\
\hline
\end{tabular}

$\mathrm{HR}=$ hazard ratio; $\mathrm{Cl}=$ confidence interval; $\mathrm{NLR}=$ neutrophil-tolymphocyte ratio. 

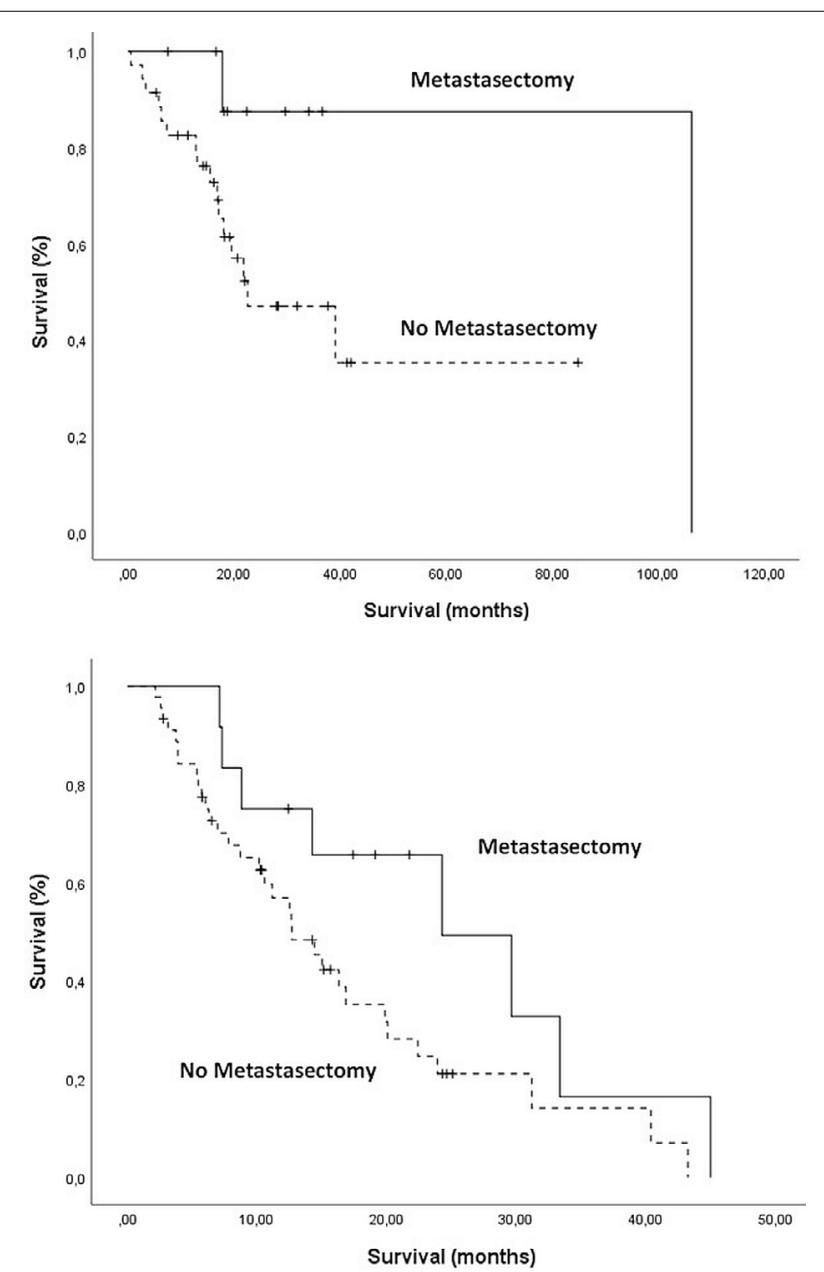

FIGURE 3 Kaplan-Meier survival analyses by risk group and history of metastasectomy. (A) In the low-risk group [neutrophil-to-lymphocyte ratio $(\mathrm{NLR})<2.35$ ], median overall survival was longer for patients receiving than not receiving metastasectomy $(p=0.05)$. (B) In the high-risk group (NLR $\geq 2.35$ ), median overall survival was nonsignificantly different for patients receiving and not receiving metastasectomy $(p=0.08)$.

\section{DISCUSSION}

This retrospective analysis of patients diagnosed and treated for mCRC provides real-world evidence about the prognostic role of the NLR, when estimated before first-line CTx.

Many factors have been proved to be useful in determining prognosis in mCRC. Patient-related (age, performance status, comorbidities), tumour-related (local growth, distant metastasis, sidedness), biochemical (markers such as carcinoembryonic antigen, lactate dehydrogenase, platelets, leucocytes, hemoglobin, alkaline phosphatase, albumin), and molecular factors (KRAS, NRAS, and $B R A F$ mutations) have all been linked to survival outcomes in $\mathrm{mCRC}^{21,22}$. Moreover, in recent decades, definitive evidence was presented establishing the association between inflammation and cancer development ${ }^{6,23}$. As a result, various studies have suggested that analysis of inflammatory factors could be helpful for predicting survival in mCRC, including assessment of inflammatory cells in peripheral blood. Because other non-cancer-related factors can also affect the systemic leucocyte count, ratios between inflammatory cells, such as the NLR, have been proposed ${ }^{24}$.

Most published analyses about the association of NLR with survival outcomes use similar NLR cut-offs, such as 2 or 5 , that usually differentiate patients into low- and highrisk groups ${ }^{15-18}$. However, in our cohort, we calculated a more accurate cut-off by analyzing the Roc curve. The 2.35 cut-off had a sensitivity of $69.0 \%$ and a specificity of $61.4 \%$. We therefore used the 2.35 value to categorize our patients into either a low-risk group $(<2.35)$ or a high-risk group ( $\geq 2.35$ ). The Kaplan-Meir estimation of mos in those risk groups revealed a statistically significant difference of 24.7 months between the low- and high-risk groups (39.1 months vs. 14.4 months). Such a difference supports the clinical usefulness of the NLR as a tool to classify patients by survival risk before delivering first-line CTx for mCRC.

Further, we used multivariate Cox regression to investigate the association of the NLR with other clinicopathologic factors. The subset of patients identified as high-risk (NLR $\geq 2.35$ ) were found to have an increase in the risk of death by approximately a factor of 3 . Those real-life results confirm that the NLR can be classified as an independent prognostic factor for poor survival in the mCRC setting. Looking at the literature, the hazard ratio associated with the NLR in our analysis is slightly higher than those in most other published results. A possible explanation for the difference is that, in our study, we used a ROC estimation to determine the NLR cut-off. Another factor that might have contributed to the hazard ratio difference is that we analyzed only patients with stage IV $\mathrm{CRC}^{12,25,26}$.

The initial choice of CTx regimen was also proved, in other reports, to be strongly associated with disease development and survival outcomes in mCRC. That effect becomes even more relevant when CTx intensification is required before ablation or surgical resection of metasta$\mathrm{ses}^{21}$. Stratification of patients based on their survival risk as prognosticated by the NLR before first-line CTx therefore provides immediate and useful data to better decide who might benefit the most from intensified systemic treatment.

Thus, taking into account the importance of CTx choice in the first-line setting, we decided to independently study the two NLR risk groups and to compare mos for patients who did and did not receive metastasectomy(by surgical or ablative intervention). In the low-risk group, the disparity in mos between patients receiving and not receiving metastasectomy reached 72 months ( $p=0.05$ ); in the high-risk group, the difference in mos was estimated to be only about 12 months $(p=0.08)$. Because only approximately $20 \%$ of patients in the low-risk group underwent resection, we believe that the reported survival difference might be statistically overestimated; nonetheless, it shows an evident tendency of patients having a low NLR, compared with those having high NLR, to achieve a greater survival benefit after metastasectomy. It is also interesting to notice that, in our cohort analysis, the mos in low-risk patients not receiving metastasectomy was similar to that in high-risk patients who did receive metastasectomy (Figure 4). Regardless, the survival benefit with the use of metastasectomy in the lowrisk group accords with results in other studies in which analysis of the NLR was performed for patients undergoing that procedure ${ }^{19}$. 


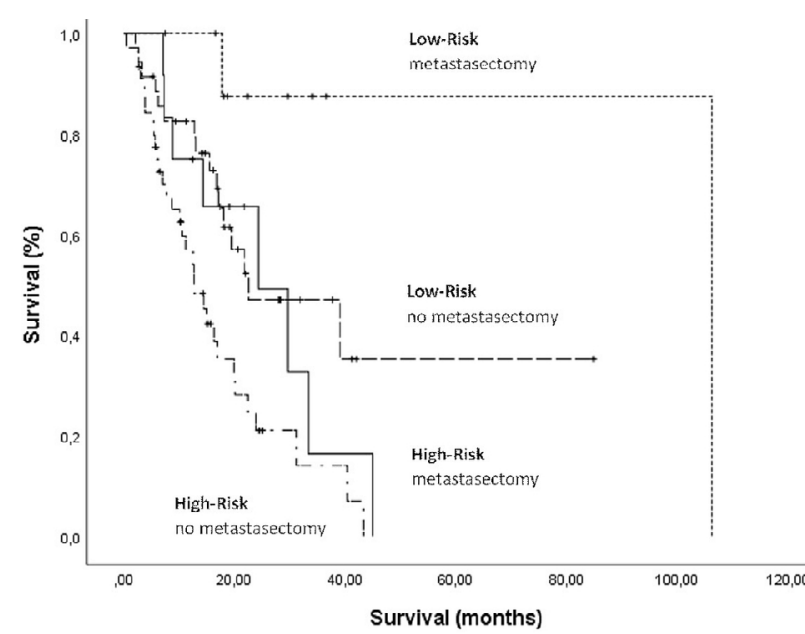

FIGURE 4 Kaplan-Meier survival analyses by risk group and history of metastasectomy combined. Median overall survival was similar for patients with low-risk disease and no metastasectomy and for those with high-risk disease who also received metastasectomy $(p=0.01)$.

A limitation of our retrospective design is that the intent of first-line CTx (conversion to resectability vs. disease control) should have been determined and investigated. By analyzing that variable, we would have clarified which patients did not undergo metastasectomy when they had been planned to receive a CTx regimen with the goal of cytoreduction. We believe that exploration of CTx intent might confirm the NLR's role as a good treatment predictor-a hypothesis that should be researched in future. In addition, characterization of the metastasis burden, metastasis locations, and types of metastasectomy were not considered in our study. We recognize that those factors could represent a relevant bias in our results and should be weighed in subsequent explorations. Additionally, only approximately $20 \%$ of our study population underwent metastasectomy, and thus the statistical analysis of such a small group might result in overestimates of effect.

Nevertheless, our study shows the clinical benefit of using the NLR as a tool for predicting survival in patients with mCRC. It also demonstrated that determining the NLR risk group before administration of first-line CTx could be useful in determining which patients might benefit the most from metastasectomy.

\section{CONCLUSIONS}

The NLR is an easily calculated tool that has been shown to accurately subcategorize patients into high- and low-risk groups in various malignancies at various stages.

The present real-world analysis showed that NLR estimation can also predict survival outcomes in patients with mCRC before first-line CTx. It also revealed that the NLR can be used as tool when deciding the intent of a CTx regimen in mCRC and whether the patient will benefit from surgical resection of metastases.

\section{CONFLICT OF INTEREST DISCLOSURES}

We have read and understood Current Oncology's policy on disclosing conflicts of interest, and we declare that we have none.

\section{AUTHOR AFFILIATIONS}

*Centro Hospitalar Barreiro-Montijo, Barreiro, Portugal.

\section{REFERENCES}

1. Ferlay J, Colombet M, Soerjomataram I, et al. Cancer incidence and mortality patterns in Europe: estimates for 40 countries and 25 major cancers in 2018. Eur J Cancer 2018;103:356-87.

2. Hu Z, Ding J, MaZ, et al. Quantitative evidence for early metastatic seeding in colorectal cancer. Nat Genet 2019;51:1113-22.

3. Barel F, Cariou M, Saliou P, et al. Histopathological factors help to predict lymph node metastases more efficiently than extra-nodal recurrences in submucosa invading pT1 colorectal cancer. Sci Rep 2019;9:8342.

4. Chen CH, Hsieh MC, Hsiao PK, Lin EK, Lu YJ, Wu SY. Tumor location is an independent predictive factor for distant metastasis and metastatic sites of rectal adenocarcinoma in patients receiving total mesorectal excision. J Cancer 2018;9:950-8.

5. Pretzsch E, Bösch F, Neumann J, et al. Mechanisms of metastasis in colorectal cancer and metastatic organotropism: hematogenous versus peritoneal spread.JOncol 2019;2019:7407190.

6. Coussens LM, Werb Z. Inflammation and cancer. Nature 2002; 420:860-7.

7. QuX, Tang Y, Hua S. Immunological approaches towards cancer and inflammation: a cross talk. Front Immunol 2018;9:563.

8. Watson J, Salisbury C, Banks J, Whiting P, Hamilton W. Predictive value of inflammatory markers for cancer diagnosis in primary care: a prospective cohort study using electronic health records. Br J Cancer 2019;120:1045-51.

9. Janakiram NB, Rao CV. The role of inflammation in colon cancer. Adv Exp Med Biol 2014;816:25-52.

10. Terzi J, Grivennikov S, Karin E, Karin M. Inflammation and colon cancer. Gastroenterology 2010;138:2101-14.

11. Dolan RD, McSorley ST, Park JH, et al. The prognostic value of systemic inflammation in patients undergoing surgery for colon cancer: comparison of composite ratios and cumulative scores. Br J Cancer 2018;119:40-51.

12. Wang F, HeW, Jiang C, etal. Prognostic value of inflammationbased scores in patients receiving radical resection for colorectal cancer. BMC Cancer 2018;18:1102.

13. Pine JK, Morris E, Hutchins GG, et al. Systemic neutrophil-to-lymphocyte ratio in colorectal cancer: the relationship to patient survival, tumour biology and local lymphocytic response to tumour. Br J Cancer 2015;113:204-11.

14. Josse JM, Cleghorn MC, Ramji KM, et al. The neutrophilto-lymphocyte ratio predicts major perioperative complications in patients undergoing colorectal surgery. Colorectal Dis 2016;18:O236-42.

15. Chua W, Charles KA, Baracos VE, Clarke SJ. Neutrophil/ lymphocyte ratio predicts chemotherapy outcomes in patients with advanced colorectal cancer. BrJCancer 2011;104:1288-95.

16. Shibutani M, Maeda K, Nagahara H, et al. A high preoperative neutrophil-to-lymphocyte ratio is associated with poor survival in patients with colorectal cancer. Anticancer Res 2013;33:3291-4.

17. Dell'Aquila E, Cremolini C, Zeppola T, et al. Prognostic and predictive role of neutrophil/lymphocytes ratio in metastatic colorectal cancer: a retrospective analysis of the TRIBE study by GONO. Ann Oncol 2018;29:924-30.

18. Argiles G, Yoshino T, Ohtsu A, et al. Prognostic value of neutrophil-to-lymphocyte ratio (NLR) on overall survival (OS), progression free survival (PFS) and disease control rate (DCR) in patients with metastatic colorectal cancer (mCRC) from the RECOURSE study [abstract 744]. JClin Oncol 2018;36: [Available online at: https://ascopubs.org/doi/10.1200/ JCO.2018.36.4_suppl.744; cited 26 August 2020]

19. Kishi Y, Kopetz S, Chun YS, Palavecino M, Abdalla EK, Vauthey JN. Blood neutrophil-to-lymphocyte ratio predicts survival 
in patients with colorectal liver metastases treated with systemic chemotherapy. Ann Surg Oncol 2009;16:614-22.

20. Yoshino T, Arnold D, Tanigushi H, etal. Pan-Asian adapted ESMO consensus guidelines for the management of patients with metastatic colorectal cancer: a JSMO-ESMO initiative endorsed by CSCO, KACO, MOS, SSO and TOS. Ann Oncol 2018;29:44-70.

21. Stein A, Bokemeyer C. How to select the optimal treatment for first line metastatic colorectal cancer. World J Gastroenterol 2014;20:899-907.

22. De Divitiis C, Nasti G, Montano M, Fisichella R, Iaffaioli RV, Berretta M. Prognostic and predictive response factors in colorectal cancer patients: between hope and reality. World J Gastroenterol 2014;20:15049-59.
23. Lu H, Ouyang $\mathrm{W}$, Huang C. Inflammation, a key event in cancer development. Mol Cancer Res 2006;4:221-33.

24. Faria SS, Fernandes PC Jr, Silva MJ, et al. The neutrophilto-lymphocyte ratio: a narrative review. eCancerMedicalScience 2016;10:702.

25. Kim JH, Lee LY, Kim HK, et al. Prognostic significance of the neutrophil-to-lymphocyte ratio and platelet-to-lymphocyte ratio in patients with stage III and IV colorectal cancer. World J Gastroenterol 2017;23:505-15.

26. He WZ, Hu WM, Kong PF, et al. Systemic neutrophil lymphocyte ratio and mismatch repair status in colorectal cancer patients: correlation and prognostic value. J Cancer 2018;9:3093-100. 